\title{
BOOLEAN DIFFERENTIATION EQUATIONS APPLICABLE IN RECONFIGURABLE COMPUTATIONAL MEDIUM
}

\author{
Stanislav Shidlovskiy ${ }^{1,2}$ \\ ${ }^{1}$ National Research Tomsk State University, 634050, Tomsk, Russia \\ ${ }^{2}$ National Research Tomsk Polytechnic University, 634050, Tomsk, Russia
}

\begin{abstract}
High performance computing environment synthesis with parallel architecture reconstructing throughout the process itself is described. Synthesized computational medium involving Boolean differential equation calculations so as to function in real-time image processing. Automaton imaging was illustrated involving the rearrangement of every processing medium element to calculate the partial differentials of $n$-th order in respect to Boolean function variables. The method of obtaining setting codes for each element was also described. An example in calculating $2^{\text {nd }}$-order Boolean derivative to two differentials in respect to Boolean functions, depending on three arguments within the reconstructible computational medium of $8 \times 8$ processing elements was given.
\end{abstract}

\section{Introduction}

Architecting updated robotic systems functioning autonomously in a-priori unknown medium involves directly positioned in- robot computer systems in order to process a considerable body of sematic information. Real-time processing of this information attributable to classical computing architecture results in increasing robot weight and structure design.

One possible solution of above-mentioned problem could be applying the reconfigurable computing medium (RCM) [1-6]. Hardware-in-the-loop RCM design and its functioning is based on grouped evaluator model:

$$
S=<C, G, A(P(D))>,
$$

where, $C=\left\{c_{i}\right\}$ - set of processing elements $c_{i}, i=0,1, \ldots, N-1 ; G$ - intercomputing linkage matrix structure (grid); $A$ - processing element algorithm set $C$ interrelated with $G$, in hardware parallel support program $P$ of data processing $D$.

Grouped evaluator construct $H=<C, G>$ reflects the following architectural concept:

1) parallelism in processing (parallel processing element algorithm set $C$ intercomputing with $G$ );

2) programmability structure;

3) homogeneity of structure $H$ (homogeneity of processing elements $c_{i} \in C$ and macrostructure $G)$. 
Let's consider the case when functioning processing element algorithm $c_{i}$ is the basis $\{\mathrm{AND}, \mathrm{OR}, \mathrm{NOT}\}$, thus acquiring the hardware invariance property.

Algorithm design in terms of solving one specific problem-oriented task via RCM, bearing on this limitation is associated with significant peformance. This paper describes the synthesis of such RCM for Boolean differentiation implementation and integration.

\section{Problem statement}

Usually, binary data is represented as $\{0,1\}$ and would look like arbitrary finite dimension matrix. By zero extension it will be representable as $2^{n} \times 2^{n}$ matrix $\mathbf{R}_{2^{n}}$, where $n$-positive integer number. This matrix $\mathbf{R}_{2^{n}}$ could be interpreted as microsequencing or code of Boolean function system, binary image (animated object frame), dynamic binary system state at the present sampling time, operating technical system component interaction graph. Further, this matrix will be considered as a system of $2^{n}$ Boolean functions (BF) $f_{j}(X)$, $j=\overline{0,2^{n}-1}, n-$ variables $x_{1}, x_{2}, \ldots, x_{n}$, Each $\mathrm{BF}$ has its own vector value $\mathbf{X}_{j}=\left[\begin{array}{lll}x_{j}^{(0)} & x_{j}^{(1)} \mathrm{K} & x_{j}^{\left(2^{n}-1\right)}\end{array}\right]^{\mathrm{T}}$, where $\mathrm{T}$ - transposition symbol; element $x_{j}^{(t)} \in(0,1)-j$-value of BF for set of variables $x_{1}^{t_{1}}, x_{2}^{t_{2}}, \ldots, x_{n}^{t_{n}} ; t_{1}, t_{2}, \ldots, t_{n}$ - binary representation parameter $t=\overline{0,2^{n}-1} ; x^{0}=\bar{x}, x^{1}=x$. The system of $2^{n} \mathrm{BF} f_{j}(X)$, having its own vector value $\mathbf{X}_{j}$, can be written in the form of the matrix

$$
\mathbf{R}_{2^{n}}=\left[\mathbf{X}_{2^{n}-1} \vdots \cdots \vdots \mathbf{X}_{1} \vdots \mathbf{X}_{0}\right] \text {. }
$$

In [7] the algorithm of Boolean derivative calculations by private variable is:

$$
\begin{array}{r}
\frac{\partial^{(k)} \mathbf{X}_{j}}{\partial x_{1} \partial x_{2} \ldots \partial x_{k}}=\mathbf{D}_{2^{n}}^{(k)} \ldots \mathbf{D}_{2^{n}}^{(2)} \mathbf{D}_{2^{n}}^{(1)} \mathbf{X}_{j}(\bmod 2), \\
\mathbf{D}_{2^{n}}^{(i)}=\mathbf{I}_{2^{i-1}} \otimes\left[\begin{array}{ll}
1 & 1 \\
1 & 1
\end{array}\right] \otimes \mathbf{I}_{2^{n-i}} .
\end{array}
$$

where I - identity matrix.

The analysis of the algorithm shows that RCM processing element should implement one of the following automaton-based display image (Figure 1).

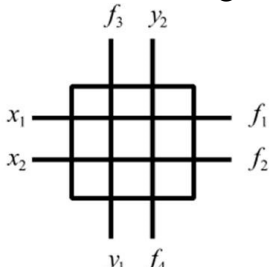

$y_{1} f_{4}$

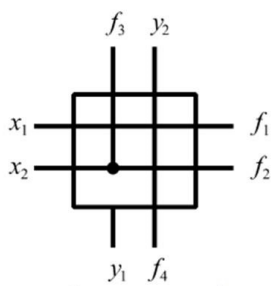

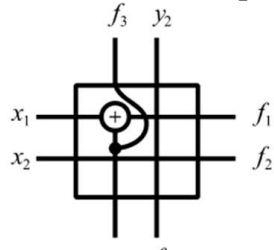

$y_{1} f_{4}$

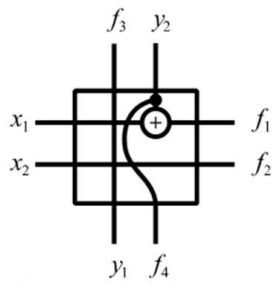

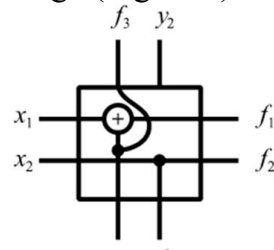

$\begin{array}{ll}y_{1} & f_{4}\end{array}$

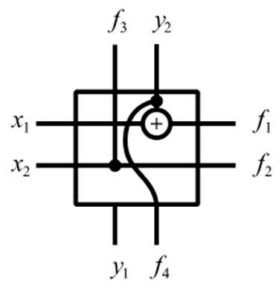

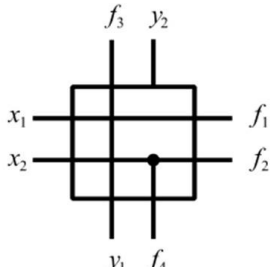

$y_{1} f_{4}$

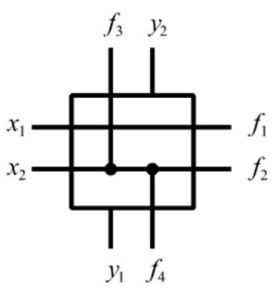

Figure 1. Automaton-based display images. 
Applying automaton-structured method [5] RCM functioning processing element algorithm could be synthesized:

$$
\left\{\begin{array}{l}
f_{1}=\left(x_{1} \bar{y}_{1} \bar{z}_{2}\right)+\left(x_{1} \bar{y}_{2} z_{2}\right)+\left(x_{1} z_{3}\right)+\left(\bar{x}_{1} y_{1} \bar{z}_{2} \bar{z}_{3}\right)+\left(\bar{x}_{1} y_{2} z_{2} \bar{z}_{3}\right) \\
f_{2}=x_{2} \\
f_{3}=\left(x_{2} z_{1} z_{2} \bar{z}_{3}\right)+\left(x_{2} z_{1} \bar{z}_{2} z_{3}\right)+\left(x_{2} \bar{z}_{1} z_{2} z_{3}\right)+\left(y_{1} z_{1} z_{2} z_{3}\right)+\left(y_{1} \bar{z}_{1} \bar{z}_{2}\right)+\left(y_{1} \bar{z}_{1} \bar{z}_{3}\right)+\left(y_{2} z_{1} \bar{z}_{2} \bar{z}_{3}\right), \\
f_{4}=\left(x_{2} z_{1} \bar{z}_{2} \bar{z}_{3}\right)+\left(x_{2} \bar{z}_{1} z_{3}\right)+\left(y_{2} z_{1} z_{2}\right)+\left(y_{2} z_{1} z_{3}\right)+\left(y_{2} \bar{z}_{1} \bar{z}_{3}\right) .
\end{array}\right.
$$

where, $x_{1}, x_{2}, y_{1}, y_{2}-$ data inputs; $f_{1}, \ldots, f_{4}-$ outputs; $z_{1}, \ldots, z_{3}$ - setup inputs of RCM processing element $[3,5]$.

Realization of algorithm (1) into RCM via evaluators, implementing automaton images (fig.1) are plotted as the matrix structure of computing-based system. Determining the matrix $Z$ configuration is based on the setting up each processing element within one possible automaton image:

$$
\begin{gathered}
\mathbf{Z}=\sum_{i} \mathbf{M}_{i} \\
\mathbf{M}_{1}=\left(\mathbf{A} \circ \mathbf{I}_{n}\right) k_{1}, \\
\mathbf{M}_{2}=\left(\mathbf{A} \circ \overline{\mathbf{I}}_{n} \circ \mathbf{H}_{n}\right) k_{2}, \\
\mathbf{M}_{3}=\left(\mathbf{A} \circ\left(\overline{\mathbf{I}}_{n} \circ \mathbf{H}_{n}\right)^{\mathrm{T}}\right) k_{3}, \\
\mathbf{M}_{4}=\overline{\mathbf{A}} k_{4}, \\
\mathbf{A}=\mathbf{D}_{2^{n}}^{(k)} \ldots \mathbf{D}_{2^{n}}^{(2)} \mathbf{D}_{2^{n}}^{(1)}(\bmod 2)
\end{gathered}
$$

where, o - Hadamard product; $\mathbf{I}_{\mathrm{n}}-n \times n$ identity matrix; $\mathbf{H}_{n}-n \times n$ upper triangle matrix of one; vertical overline $\left(^{-}\right)$means $\mathrm{x}$-element inversion in binary matrix; ${ }^{\mathrm{T}}-$ transposition matrix symbol; $k_{i}$ - automaton image code.

\section{Example}

It is required to calculate Boolean second-order derivative with respect to the $\mathrm{BF}$ variables $x_{1}$ and $x_{2}$, as in the following expression

$$
f_{i}(\mathbf{X})=\mathrm{x}_{1} x_{2} \vee x_{3} .
$$

To implement Boolean derivative calculation by private variable four basic evaluator elements are necessary (fig. 1). Each element is denoted by $k_{i}(i=1,2, \ldots, 4)$. Value $k_{i}$ corresponds to each automaton code of its configuration, introduced as decimal numeration (as follows $k_{1}=2 ; k_{2}=3, k_{3}=4, k_{4}=5$ ). We apply in (5) all possible argument values:

$$
\mathbf{X}_{j}=\left[\begin{array}{llllllll}
0 & 1 & 0 & 1 & 0 & 1 & 1 & 1
\end{array}\right]^{\mathrm{T}} .
$$

According to (3) and (4) the following can be obtained:

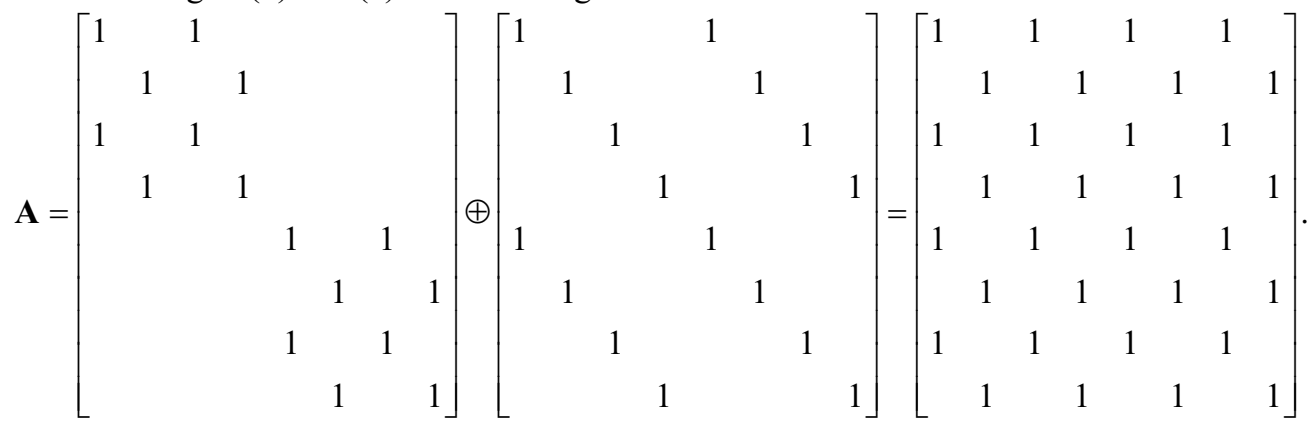




$$
\mathbf{Z}=\left[\begin{array}{llllllll}
2 & 5 & 3 & 5 & 3 & 5 & 3 & 5 \\
5 & 2 & 5 & 3 & 5 & 3 & 5 & 3 \\
4 & 5 & 2 & 5 & 3 & 5 & 3 & 5 \\
5 & 4 & 5 & 2 & 5 & 3 & 5 & 3 \\
4 & 5 & 4 & 5 & 2 & 5 & 3 & 5 \\
5 & 4 & 5 & 4 & 5 & 2 & 5 & 3 \\
4 & 5 & 4 & 5 & 4 & 5 & 2 & 5 \\
5 & 4 & 5 & 4 & 5 & 4 & 5 & 2
\end{array}\right]
$$

Obtained matrix of $Z$ configuration corresponds to RCM, depicted in Figure 2.

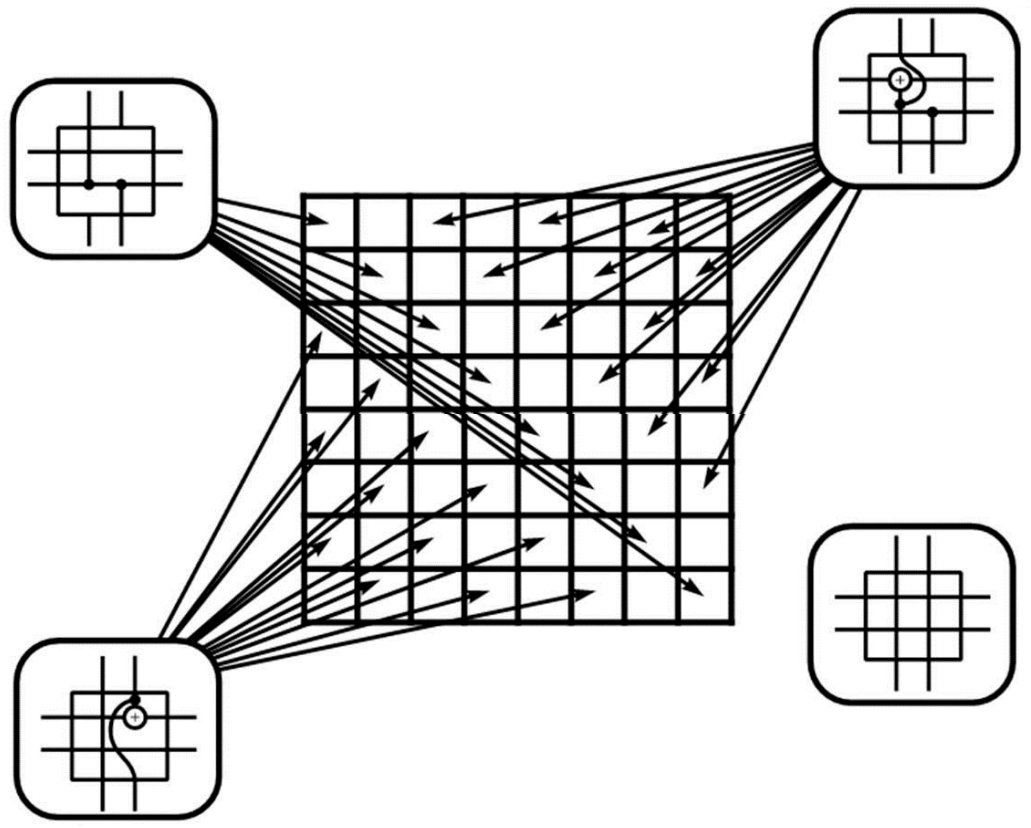

Figure 2. RCM configuration.

Having generated the signals corresponding to (6) the result providing the $\mathrm{BF}$ calculation by private variables (5) $x_{1}$ and $x_{2}$ is recorded on data inputs of obtained RCM and on medium return:

$$
\frac{\partial^{2} \mathbf{X}_{j}}{\partial x_{1} \partial x_{2}}=\left[\begin{array}{llllllll}
1 & 0 & 1 & 0 & 1 & 0 & 1 & 0
\end{array}\right]^{\mathrm{T}} .
$$

\section{Conclusion}

$\mathrm{RCM}$ algorithms were synthesized to provide the calculation of BF private variables. These algorithms meet the specified requirements applicable for VLSI process. In this case, such algorithms involve regular and localized computation communication, oriented on pipeline computations. Proposed algorithm configuration (3), involving specific setting code inputs for each RCM processing element, is responsible of calculating Boolean derivatives to different argumants within one and the same RCM.

According to the reference description, the synthesis method of specific Boolean function evaluators is based on the operating diagram of the previously selected Boolean 
base (AND, OR, NOT) and is housed in synthesized-based medium. It should be noted that the prescribed functional diagram or logical network within selected medium is 'fitted'. Obtained algorithms of operating RCM (reconstructible computational medium) could be the basis of developing special-purpose evaluators producing basic operations of morphologically processing digital images.

\section{Acknowledgment}

The following research has been financially supported by Russian Foundation for Basic Research (RFBR) 16-07-01138 A "Intelligent Reconfigurable Control Systems, Navigation and Image Processing for Autonomous Mobile Robots."

\section{References}

[1] I. Kulagin, A. Paznikov, M. Kurnosov, Lecture Notes in Computer Science 9251, 405 (2015) doi: 10.1007/978-3-319-21909-7_39

[2] N. Maa, Sh. Wang, Ali S. Mohsin, X. Cui, Yu. Peng, MATEC Web of Conferences 45, 05001 (2016) doi: 10.1051/matecconf/20164505001

[3] S.V . Shidlovskii, J. Comp. Sys. Sci. Inter. 45, 282 (2006) doi: 10.1134/S1064230706020122.

[4] A.I. Kalyaev, I.A. Kalyaev, J. Comp. Sys. Sci. Inter. 55, 211 (2016) doi: $10.1134 / \mathrm{S} 1064230716010081$

[5] I.A. Kalyaev, I.I. Levin, A.I. Dordopulo, L.M. Slasten, IFAC Proceedings Volumes 46, 210 (2013) doi: 10.3182/20130925-3-CZ-3023.00009

[6] D.V. Shashev, S.V. Shidlovskiy, Optoelectronics, Instrumentation and Data Processing 51, 227 (2015) doi: 10.3103/S8756699015030036

[7] S. Janushkevich, D. Bokhmann, R. Stankovich, G. Tosic, V. Shmerko, Avtomatika I Telemeknanika (Journal of Automation \& Remote Control) 6, 155 (2004). 